\title{
Pelvic Floor Function in the Female
}

\author{
Christos E. Constantinou \\ Stanford University Medical School, Stanford, USA \\ Email: chris.constantinou@stanfordalumni.org
}

Received 18 July 2014; revised 15 August 2014; accepted 8 September 2014

Copyright (C) 2014 by author and Scientific Research Publishing Inc.

This work is licensed under the Creative Commons Attribution International License (CC BY).

http://creativecommons.org/licenses/by/4.0/

(c) (i) Open Access

\section{Introduction}

Pelvic Floor (PF) disorders, such as urinary incontinence and prolapse accounted for over 400,000 operations in the USA in 1987 [1], and nearly a third of these were re-operations. It is estimated that $30 \%-50 \%$ of women in Europe and the USA are affected by Urinary incontinence [2]. Stress Urinary Incontinence (SUI), the involuntary leakage of urine on coughing, sneezing, exertion or effort, is the most common form of urinary incontinence in women. The scientific understanding of normal PF function is limited and consequently treatment of these prevalent, disabling conditions is, at best, inefficient.

Due to the location of the PF defining its normal function is challenging. The PF is under neural control and is a complex 3D arrangement of muscle and connective tissue, attached to the bony pelvis. The PFM is a collective name for the the levator ani and ischiococcygeus. The levator ani muscle consists of the pubococcygeus, the puborectalis, and the iliococcygeus muscles. The pubococcygeus and the puborectalis muscles form a U-shape as they originate from the pubic bone on either side of the midline and pass behind the rectum to form a sling. The iliococcygeus muscle arises laterally from the arcus tendineus levator ani and forms a horizontal sheet that spans the opening in the posterior region of the pelvis, thereby providing a "shelf" upon which the pelvic organs rest [3]. The muscles and fascias of the pelvic diaphragm are inserted on the ischial spines either directly or indirectly through the sacrospinous ligament and the tendinous arch of the pelvic fascia. The result of a PFM contraction is a medial pull on the ischial spines to produce a more rigid and narrower PF [4].

Many measurement tools for PFM function quantify strength but PFM strengthening is not the only intervention used clinically in rehabilitation of patients with PFM disorders. There are many methods of measuring PFM activity such as palpation, visual observation, electromyography, dynamometers, ultrasound, and magnetic resonance imaging (MRI). Each tool has its own qualities and limitations [5]. Most recently, using a reliable instrumented speculum, incontinent women demonstrated lower values in passive force, endurance and speed of contraction than continent women ( $p<0.05$ ), however, differences between the two groups for maximal force reached the statistically significant level only in the endurance parameter [6]. PFM strengthening exercises do diminish the symptoms of SUI yet a number of studies have demonstrated that strength of PFM contraction does not always correlate to continence state or action on the urethra [6]-[8], so what is it about PFM rehabilitation that helps? Little research has focused upon the mechanisms of therapeutic change to help identify the specific critical muscle components of training [9] so it is unknown whether PFM training mimics the normal physiological behavior of the PFM or is a compensation strategy, nor whether a strengthening program is indeed the most efficient method of conservative rehabilitation. It seems appropriate to determine whether other properties 
of muscle function are also important in defining PFM function and dysfunction, as well as gaining a greater understanding of why PFM rehabilitation in women with SUI is effective.

Previous visualization studies using ultrasound or MRI show that a voluntary contraction of the Pelvic Floor Muscles (PFM) changes the ano-rectal angle (ARA) [10] and can displace the urethra in a direction towards the pubic symphysis [11]-[13]. Yet why does a correct PFM contraction in some women increase the intra urethral pressure, but in others it does not [14]? It is known that in continent women there is recruitment of PFM motor units [15] and an increase in intra-urethral pressure [16] prior to an increase in intra-abdominal pressure (IAP) during a cough. Is the increase in intra-urethral pressure caused by an automatic, pre programmed activation of the PFM? If so, is this pre-activation lost or delayed in SUI? Certainly there are altered PFM activation patterns during a cough, measured by EMG in women with SUI compared to healthy volunteers [17], with shorter activation periods, lack of response or paradoxical inhibition, so how does this alteration affect the trajectory of urogenital structures?

Visualization studies have described the displacement of the urethra or bladder neck that occurs from the start to finishing point of a cough, a PFM contraction and valsalva maneuver, yet none so far have either mapped the trajectory throughout the maneuver, or defined the automatic function of the PFM, especially in response to sudden rises in intra-abdominal pressure (IAP) during a cough. Are the differences in women with PF disorders just a difference of amplitude of displacement, or are the specifics of the journey important too?

The PFM, along with the diaphragm and abdominal muscles are also thought to contribute in generating intraabdominal pressure (IAP). Disorders of breathing and incontinence have a strong association with low back pain (LBP) [18] and subjects with LBP generate more IAP during low load tasks and have alterations in the timing of trunk muscle activity [19] [20] than those without LBP. Small studies of continent women have shown that the Abdominal muscles and PFM co-activate [21] [22], is this true for a larger group size, and are there any differences in women with SUI or other PF disorders?

To answer those important clinical questions, it is essential to establish reliable methods for the evaluation of the PF functions. The reliable methods to evaluate the changes of the PF functions will be helpful for the physicians or clinicians to identify those patients who are likely to be rehabilitated by PF exercises instead of by surgery.

\section{Current Methods Used in Clinics}

In current clinical practice, manual muscle testing per vagina is the technique used by most clinicians to evaluate a PFM contraction using a 5 point modified Oxford Grading ordinal scale [23]. It can measure whether or not there is a correct contraction and the strength of it. The main limitation of this method is the length of time the assessment takes and the ease with which most clinicians, other than physiotherapists, could incorporate it into their clinical practice. Vaginal palpation has also been criticized for its lack of reliability and sensitivity in the measurement of pelvic floor muscles strength for scientific purposes [24]. Because of the inherent limitations of vaginal palpation, other methods such as vaginal cones, hydrostatic pressure, surface, needle and wire EMG are currently tested to assess PF function and the improvement of PF function in conservative treatment. The clinical applications of those methods are limited because they are invasive and inconvenient to be applied in regular clinical examination.

2D ultrasound imaging can acquire dynamic information on the morphology of the urogenital organs. In particular, perineal, introital and trans-vaginal ultrasound has become an imaging platform for the evaluation of the $\mathrm{PF}$ and for the treatment planning of many uro-gynecological conditions [24]. By its nature, 2D ultrasound imaging provides a very large amount of dynamic data that cannot be visually assimilated by the observer in its totality, particularly during fast occurring events. Such dynamic events contain information relating the integrity of the supporting structures of the bladder neck, the role of the PF, and the compliance of pelvic floor structures [25]. Furthermore, because the urogenital structures are anatomically interconnected, ultrasound-based dynamic imaging can substantiate urodynamic observations of the effective distribution of pressure transmission to the urethra [7] [16].

State-of-the-art 3D ultrasound imaging techniques provide 3D visualization of the pelvic floor structures with higher resolution. However, current 3D ultrasound machines are not fast enough for the purpose of visualization the movement of tissues in fast and stressful maneuvers like coughing, which may provoke urinary incontinence. Besides, as 3D imaging is cost prohibitive for the majority of clinicians it is anticipated that 2D ultrasound will 
be, for the foreseeable future, the measuring and feedback tool of clinical choice.

MRI imaging was introduced to study the anatomical components of the PF and thereby contribute to better understand mechanism of continence [26]-[29]. Different methods have been applied to process and analyze the MRI image to acquire the 2D anatomy of the PF with higher spatial resolution and speed of image acquisition [30]-[32]. MRI imaging in 3D, reported in the recent research literature, was achieved by reconstructing sequential 2D images acquired in the axial and sagittal plane [33] [34]. While 3D MRI imaging provides very useful insight into the anatomical configuration, the amount of time required to acquire 3D volume data is too long to record any dynamic information of the PF activities. Therefore, current 3D MRI studies are limited to analyze the passive response of the PF under a certain loading or straining [29]. MRI imaging is also too expensive to be used in regular clinical examination.

\section{Evaluation of the Dynamic Function of the PF Using 2D Ultrasound Imaging}

In real time ultrasound imaging, the diagnostically important information of the dynamic response of the PF cannot be assimilated and quantified by the observer during the scanning process. The trajectories and the timings of the movement of the PF tissues, which may be more important than the amplitudes in the mechanism of female urinary continence, are usually ignored. Current quantitative measurements of 2D ultrasound images can only tell us about the resting position of the urethra and the displacement at the end of events such as Valsalva, voluntary PFM contraction and coughing. [35]-[37]. The difficulties with accurately determining the finishing point of any maneuver, particularly those that are fast, such as coughing, are numerous and are a potential source of error [38]. The operator has either had to make multiple on-screen measurements, or determine the exact peak moment, or end position of the maneuver, freeze it, and then measure the change in position manually on screen or within in built electronic calipers. Without correcting for probe movement relative to the pubis symphysis the percentage errors range from $18 \%$ - 87\% [39]. In addition measuring only the re-positioning of urogenital structures appears to be of limited value because intermediary anatomical changes are not registered.

In order to develop more sensitive measures to define normal PF function pilot studies were performed to capture and visualize the sequence of dynamic changes the PFM produced on the urethra, vagina and rectum using digital image-processing methods. The approach taken is to use an edge extraction algorithm to outline the coordinates of the symphysis, urethra and rectum interfaces on a frame-by-frame basis for sequences of stress inducing events such a cough, Valsalva and voluntarily induced PF contractions. During each event, the trajectory of the boundary of each structure was identified to characterize the sequential history of the ensuing movement. The resulting image analysis focused to reveal the anatomical displacement of the urogenital structures and to enable the evaluation of their biomechanical parameters in terms of displacement, velocity and acceleration. On the basis of these observations, we expect that the urogenital response to PF contractions can be quantified and the mechanism of UI, including the trajectories and timing of the PF activities, can be elucidated. Given that ultrasound imaging contains a considerable amount of useful data which can be obtained with the minimum of invasion to the patient, it is appropriate to find a way that this information be revealed and displayed quantitatively.

On the basis of those pilot studies we were able to quantitatively measure, analyze and describe the whole of the movement during a particular maneuver, and are therefore able to better define new parameters of PF function. We have accounted for movement of the probe, relative to the structures being examined and do not rely upon the operator to capture the precise moment at the end of the maneuver. Confidence is required none the less to validate the ultrasound approach with subjects of a broad age group and disposition. Ultimately we believe that these visualizations, together with data obtained from the vaginal biosensor currently under study in our lab, to generate a greater understanding of the mechanisms of normal PF function and dysfunction within the groups being studied. The visualization of the PF activities using 2D ultrasound imaging is likely to develop new measures of the PF functions that are more sensitive and specific than current methods. Those measures are useful for categorizing different sub groups of patients within a particular pathology and determining the most appropriate treatment intervention.

In this research application we propose to apply 2D digital image processing, animation technology and artificial neural networks to perform the real time non-invasive ultrasound evaluations of the female PF. It is expected that upon completion of this the project, the mechanism of PF function can be better identified and more sensitive clinical diagnoses and treatment outcome measures for SUI can be developed. In the future, it is antic- 
ipated that the technology developed will be applicable to study other groups with PF dysfunctions, such as prolapse, pelvic pain, vulvodynia and sexual dysfunction.

Clearly there are multiple technical and clinical rationales in our approach justifying the conceptual framework proposed at this particular time given the significance of the project. Clinically it is evident that millions of older women around the world will tackle incontinence with its medical and lifestyle-altering sequels. Systematic approach is therefore fundamental in the development of potentially effective interventions, and in so doing enabling the identification of suitable candidates likely to respond to training, surgery or other yet to be devised regimens. Considering the number of women who will be entering their sixties over the next few generations and the losses in time, expenses and resources directed towards badly targeted interventions, the need is compelling for a model that will enable a reliable distinction to be made between those women who will respond to training and those who will not, becoming disillusioned. We suggest our approach, using innovative graphics, could be an effective demonstration to both physicians and patients to illustrate the importance of visualization to existing pro continence processes such as pre-contraction and other anticipatory maneuvers. Ultimately it is reasonable to be able to predict which is the appropriate existing treatment that can be specifically directed, and inevitably in which direction to search for new non empirical approaches to be innovated by the clinical investigators.

In addition to generating the evidence to test the proposed hypotheses we suggest that it may be of importance to disseminate to the medical Community, the results of these studies subsequent to peer scrutiny. Once diffused, they should contribute to the medical and scientific community as a whole, by providing better understanding of normal and pathological PFM morphology and function in older women in addition to a better understanding of the morphological causes of PFM functional abnormality. Moreover, the results of this research program will provide clinicians with a new low-cost PFM training program to treat UI in older women as well as the capacity to identify those who will benefit from PFM training.

\section{References}

[1] DeLancey, J.O. (2005) The Hidden Epidemic of Pelvic Floor Dysfunction: Achievable Goals for Improved Prevention and Treatment. American Journal of Obstetrics \& Gynecology, 192, 1488-1495. http://dx.doi.org/10.1016/j.ajog.2005.02.028

[2] Buchsbaum, G.M., Duecy, E.E., et al. (2005) Urinary Incontinence in Nulliparous Women and Their Parous Sisters. Obstetrics Gynecology, 106, 1253-1258. http://dx.doi.org/10.1097/01.AOG.0000187309.46650.b2

[3] DeLancey, J.O., Strohbehn, K., et al. (1998) Comparison of Ureteral and Cervical Descents during Vaginal Hysterectomy for Uterine Prolapse. American Journal of Obstetrics \& Gynecology, 179, 1405-1410. http://dx.doi.org/10.1016/S0002-9378(98)70002-X

[4] Abitbol, M.M. (1988) Evolution of the Ischial Spine and of the Pelvic Floor in the Hominoidea. American Journal of Physical Anthropology, 75, 53-67. http://dx.doi.org/10.1002/ajpa.1330750107

[5] Bo, K. and Sherburn, M. (2005) Evaluation of Female Pelvic-Floor Muscle Function and Strength. Physical Therapy, 85, 269-282.

[6] Morin, M., Bourbonnais, D., et al. (2004) Pelvic Floor Muscle Function in Continent and Stress Urinary Incontinent Women Using Dynamometric Measurements. Neurourology and Urodynamics, 23, 668-674. http://dx.doi.org/10.1002/nau.20069

[7] Kessler, R. and Constantinou, C.E. (1986) Internal Urethrotomy in Girls and Its Impact on the Urethral Intrinsic and Extrinsic Continence Mechanisms. The Journal of Urology, 136, 1248-1253.

[8] Theofrastous, J.P., Wyman, J.F., et al. (2002) Effects of Pelvic Floor Muscle Training on Strength and Predictors of Response in the Treatment of Urinary Incontinence. Neurourology and Urodynamics, 21, 486-490. http://dx.doi.org/10.1002/nau.10021

[9] Nygaard, I. (2004) Physiologic Outcome Measures for Urinary Incontinence. Gastroenterology, 126, S99-S105. http://dx.doi.org/10.1053/j.gastro.2003.10.009

[10] Costantini, S., Esposito, F., et al. (2006) Ultrasound Imaging of the Female Perineum: The Effect of Vaginal Delivery on Pelvic Floor Dynamics. Ultrasound in Obstetrics Gynecology, 27, 183-187. http://dx.doi.org/10.1002/uog.2663

[11] Miller, J.M., Perucchini, D., et al. (2001) Pelvic Floor Muscle Contraction during a Cough and Decreased Vesical Neck Mobility. Obstetrics Gynecology, 97, 255-260. http://dx.doi.org/10.1016/S0029-7844(00)01132-7

[12] Constantinou, C.E., Hvistendahl, G., et al. (2002) Determining the Displacement of the Pelvic Floor and Pelvic Organs during Voluntary Contractions Using Magnetic Resonance Imaging in Younger and Older Women. BJU International, 
90, 408-414. http://dx.doi.org/10.1046/j.1464-410X.2002.02907.x

[13] Dietz, H.P. (2004) Ultrasound Imaging of the Pelvic Floor. Part II: Three-Dimensional or Volume Imaging. Ultrasound in Obstetrics Gynecology, 23, 615-625. http://dx.doi.org/10.1002/uog.1072

[14] Bump, R.C., Hurt, W.G., et al. (1991) Assessment of Kegel Pelvic Muscle Exercise Performance after Brief Verbal Instruction. American Journal of Obstetrics Gynecology, 165, 322-329. http://dx.doi.org/10.1016/0002-9378(91)90085-6

[15] Deindl, F.M., Vodusek, D.B., et al. (1993) Activity Patterns of Pubococcygeal Muscles in Nulliparous Continent Women. British Journal of Urology, 72, 46-51. http://dx.doi.org/10.1111/j.1464-410X.1993.tb06455.x

[16] Constantinou, C.E. and Govan, D.E. (1982) Spatial Distribution and Timing of Transmitted and Reflexly Generated Urethral Pressures in Healthy Women. Journal of Urology, 127, 964-969.

[17] Deindl, F.M., Vodusek, D.B., et al. (1994) Pelvic Floor Activity Patterns: Comparison of Nulliparous Continent and Parous Urinary Stress Incontinent Women. A Kinesiological EMG Study. British Journal of Urology, 73, $413-417$. http://dx.doi.org/10.1111/j.1464-410X.1994.tb07606.x

[18] Smith, M.D., Russell, A., et al. (2006) Disorders of Breathing and Continence Have a Stronger Association with Back Pain than Obesity and Physical Activity. Australian Journal of Physiotherapy, 52, 11-16. http://dx.doi.org/10.1016/S0004-9514(06)70057-5

[19] Hodges, P.W. and Richardson, C.A. (1999) Altered Trunk Muscle Recruitment in People with Low Back Pain with Upper Limb Movement at Different Speeds. Archives of Physical Medicine and Rehabilitation, 80, 1005-1012. http://dx.doi.org/10.1016/S0003-9993(99)90052-7

[20] Ferreira, P.H., Ferreira, M.L., et al. (2004) Changes in Recruitment of the Abdominal Muscles in People with Low Back Pain: Ultrasound Measurement of Muscle Activity. Spine, 29, 2560-2566. http://dx.doi.org/10.1097/01.brs.0000144410.89182.f9

[21] Sapsford, R.R., Hodges, P.W., et al. (2001) Co-Activation of the Abdominal and Pelvic Floor Muscles during Voluntary Exercises. Neurourology and Urodynamics, 20, 31-42. http://dx.doi.org/10.1002/1520-6777(2001)20:1<31::AID-NAU5>3.0.CO;2-P

[22] Neumann, P. and Gill, V. (2002) Pelvic Floor and Abdominal Muscle Interaction: EMG Activity and Intra-Abdominal Pressure. International Urogynecology Journal and Pelvic Floor Dysfunction, 13, 125-132. http://dx.doi.org/10.1007/s001920200027

[23] Laycock, J., Brown, J., et al. (2001) Pelvic Floor Reeducation for Stress Incontinence: Comparing Three Methods. British Journal of Community Nursing, 6, 230-237. http://dx.doi.org/10.12968/bjcn.2001.6.5.7083

[24] Bo, K., Lilleas, F., et al. (2001) Dynamic MRI of the Pelvic Floor Muscles in an Upright Sitting Position. Neurourology and Urodynamics, 20, 167-174. http://dx.doi.org/10.1002/1520-6777(2001)20:2<167::AID-NAU19>3.0.CO;2-4

[25] Barbic, M., Kralj, B., et al. (2003) Compliance of the Bladder Neck Supporting Structures: Importance of Activity Pattern of Levator Ani Muscle and Content of Elastic Fibers of Endopelvic Fascia. Neurourology and Urodynamics, 22, 269-276. http://dx.doi.org/10.1002/nau.10116

[26] Klutke, C., Golomb, J., et al. (1990) The Anatomy of Stress Incontinence: Magnetic Resonance Imaging of the Female Bladder Neck and Urethra. Journal of Urology, 143, 563-566.

[27] Yang, A., Mostwin, J.L., et al. (1991) Pelvic Floor Descent in Women: Dynamic Evaluation with Fast MR Imaging and Cinematic Display. Radiology, 179, 25-33. http://dx.doi.org/10.1148/radiology.179.1.2006286

[28] Christensen, L.L., Djurhuus, J.C., et al. (1995) Imaging of Pelvic Floor Contractions Using MRI. Neurourology and Urodynamics, 14, 209-216. http://dx.doi.org/10.1002/nau.1930140302

[29] Stoker, J., Halligan, S., et al. (2001) Pelvic Floor Imaging. Radiology, 218, 621-641. http://dx.doi.org/10.1148/radiology.218.3.r01mr26621

[30] Comiter, C.V., Vasavada, S.P., et al. (1999) Grading Pelvic Prolapse and Pelvic Floor Relaxation Using Dynamic Magnetic Resonance Imaging. Urology, 54, 454-457. http://dx.doi.org/10.1016/S0090-4295(99)00165-X

[31] Goh, V., Halligan, S., et al. (2000) Dynamic MR Imaging of the Pelvic Floor in Asymptomatic Subjects. American Journal of Roentgenology, 174, 661-666. http://dx.doi.org/10.2214/ajr.174.3.1740661

[32] Gousse, A.E., Barbaric, Z.L., et al. (2000) Dynamic Half Fourier Acquisition, Single Shot Turbo Spin-Echo Magnetic Resonance Imaging for Evaluating the Female Pelvis. Journal of Urology, 164, 1606-1613. http://dx.doi.org/10.1016/S0022-5347(05)67040-1

[33] Athanasiou, S., Khullar, V., et al. (1999) Imaging the Urethral Sphincter with Three-Dimensional Ultrasound. Obstetrics Gynecology, 94, 295-301. http://dx.doi.org/10.1016/S0029-7844(99)00247-1

[34] Fielding, J.R., Dumanli, H., et al. (2000) MR-Based Three-Dimensional Modeling of the Normal Pelvic Floor in Women: Quantification of Muscle Mass. American Journal of Roentgenology, 174, 657-660. 
http://dx.doi.org/10.1016/S0029-7844(99)00247-1

[35] Schaer, G.N., Perucchini, D., et al. (1999) Sonographic Evaluation of the Bladder Neck in Continent and Stress-Incontinent Women. Obstetrics Gynecology, 93, 412-416. http://dx.doi.org/10.1016/S0029-7844(98)00420-7

[36] Howard, D., Miller, J.M., et al. (2000) Differential Effects of Cough, Valsalva and Continence Status on Vesical Neck Movement. Obstetrics Gynecology, 95, 535-540. http://dx.doi.org/10.1016/S0029-7844(98)00420-7

[37] Dietz, H.P., Jarvis, S.K., et al. (2002) The Assessment of Levator Muscle Strength: A Validation of Three Ultrasound Techniques. International Urogynecology Journal and Pelvic Floor Dysfunction, 13, 156-159. http://dx.doi.org/10.1007/s192-002-8346-5

[38] Pregazzi, R., Sartore, A., et al. (2002) Perineal Ultrasound Evaluation of Urethral Angle and Bladder Neck Mobility in Women with Stress Urinary Incontinence. BJOG, 109, 821-827. http://dx.doi.org/10.1111/j.1471-0528.2002.01163.x

[39] Reddy, A.P., DeLancey, J.O., et al. (2001) On-Screen Vector-Based Ultrasound Assessment of Vesical Neck Movement. American Journal of Obstetrics Gynecology, 185, 65-70. http://dx.doi.org/10.1067/mob.2001.116373 
Scientific Research Publishing (SCIRP) is one of the largest Open Access journal publishers. It is currently publishing more than 200 open access, online, peer-reviewed journals covering a wide range of academic disciplines. SCIRP serves the worldwide academic communities and contributes to the progress and application of science with its publication.

Other selected journals from SCIRP are listed as below. Submit your manuscript to us via either submit@scirp.org or Online Submission Portal.
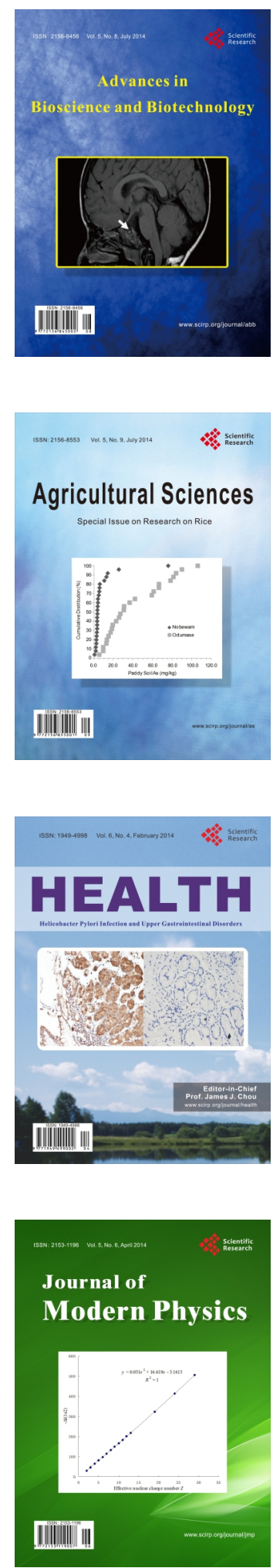
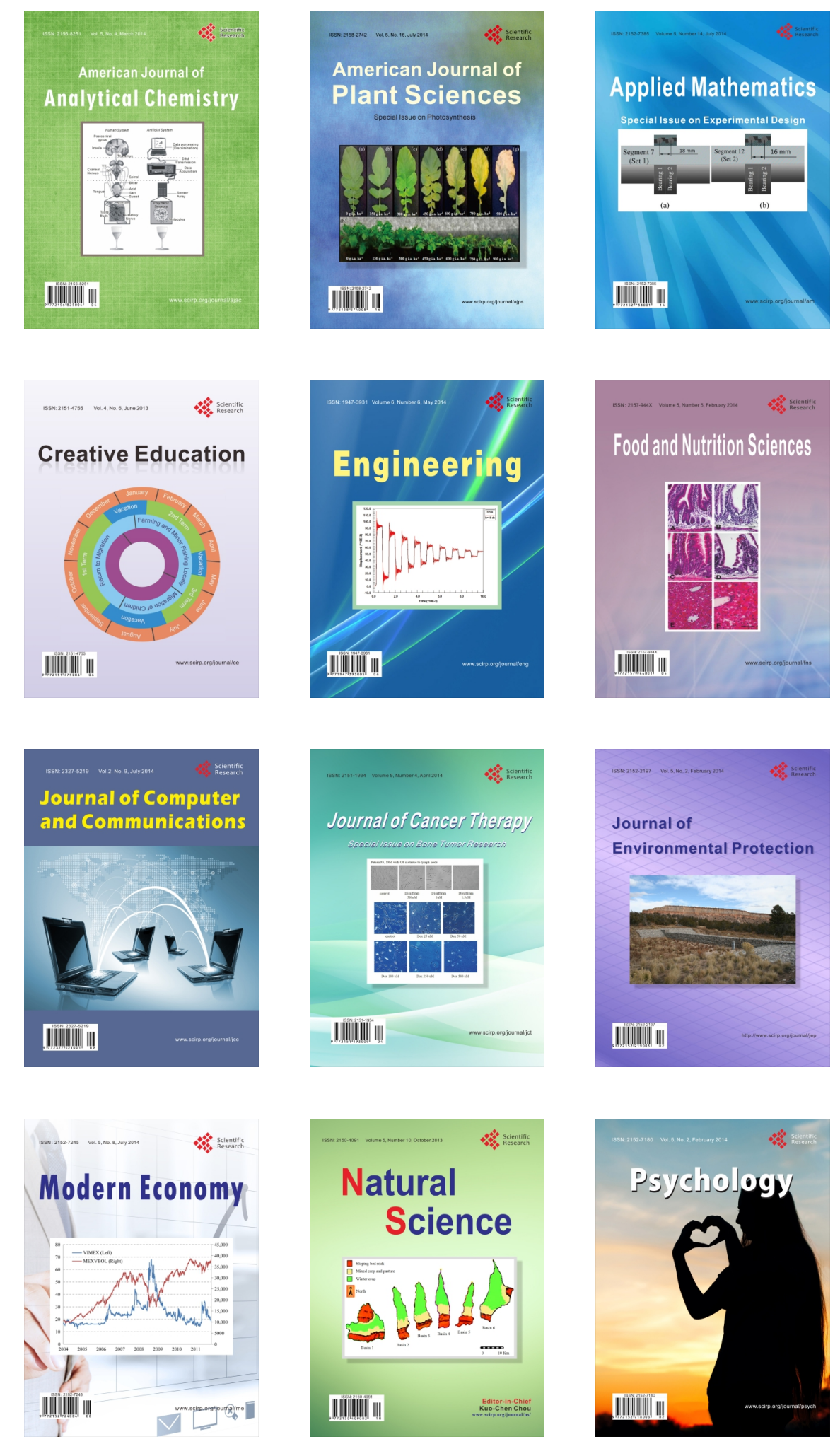